\title{
UNCERTAINTIES ON THE LUMINESCENCE AGES AND ANOMALOUS FADING
}

\author{
ANTOINE ZINK \\ Centre de Recherche et de Restauration des Musées de France, Palais du Louvre, Porte des lions \\ 14 quai F. Mitterrand, 75001 Paris
}

Received 15 July 2008

Accepted 2 December 2008

\begin{abstract}
It is well known that some minerals give underestimated luminescence ages due to anomalous fading. The anomalous fading follows a logarithmic decay law characterized by its slope, the socalled fading rate or g-value. Using the fading rate, Huntley and Lamothe (2001) suggested some correction for the fading underestimation of young samples $(<40-50 \mathrm{ka})$. For polymineral fine grains, we observe a fading rate of $0-4 \% /$ decade for TL and BL-OSL and $4-6 \% /$ decade for IR-OSL. Extending the laboratory observation to archaeological age, the underestimation on the age for $10 \mathrm{ka}$ is estimated to a mean of $5 \%$ for TL, $10 \%$ for BL-OSL and $45 \%$ for IR-OSL. Due to the non-linearity of the Huntley and Lamothe's fading correction, the contribution of the fading to the total uncertainty is estimated by a Monte-Carlo simulation. The inference on dating shows that the uncertainty on the anomalous fading can be a significant term of the combined uncertainty on the age, even for low fading rates.
\end{abstract}

Keywords: Luminescence dating, anomalous fading, uncertainties.

\section{INTRODUCTION}

It is well known that some minerals (e.g. feldspar) give underestimated luminescence ages due to the anomalous fading (Wintle, 1973). The anomalous fading follows a logarithmic decay law. The slope of the decay law, called fading rate, can vary from 0 to ca. $20 \%$ decade of time (Visocekas, 1988).

Using the fading rate, Huntley and Lamothe (2001) suggested some correction for the fading underestimation of the young age $(<40-50 \mathrm{ka})$. From their investigation, the anomalous fading appeared to be ubiquitous. However, Preusser (2003) denied the ubiquity of the anomalous fading on feldspars. Our knowledge on the fading rate is limited by the limit of detection of the fading rate, close to $0.6 \%$ /decade using the SAR fading protocol as suggested by Auclair et al. (2003). It is then necessary to estimate the underestimation introduced by low fading rate and the uncertainty contribution of the anomalous fading.

This paper is based on a collection of our own measurements made by SAR fading protocol on polymineral fine grain samples since 2004. Section 2 presents labora-

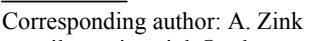

e-mail: antoine.zink@culture.gouv.fr

ISSN 1897-1695 (online), 1733-8387 (print) (C) 2008 GADAM Centre, Institute of Physics, Silesian University of Technology.

All rights reserved. tory conditions and the distribution of g-values for TL and OSL measurements. Section 3 deals with the error introduced by the anomalous fading. The range of underestimation is obtained by extending the luminescence loss to archaeological duration (long-term fading) using previous g-values (section 2). Section 4 gives a method to estimate the contribution of the uncertainty on the correction of the anomalous fading by the use of a Monte Carlo simulation.

\section{MEASUREMENTS}

\section{Equipments}

All measurements were made on the $4-13 \mu \mathrm{m}$ polymineral fraction. The luminescence measurements were performed with a Risoe TL/OSL DA-15 equipped with an EMI 9235QA PMT and incorporated ${ }^{90} \mathrm{Sr} /{ }^{90} \mathrm{Y}$ source (7.4 Gy/min on 1st January 2005). For optical stimulation, we used infrared laser diode $(830 \pm 10 \mathrm{~nm} ; 50 \%$ of $450 \mathrm{~mW} / \mathrm{cm}^{2}$ full power - IR-OSL) or 21 pairs of blue diodes $\left(470 \pm 30 \mathrm{~nm} ; 50 \%\right.$ of $19 \mathrm{~mW} / \mathrm{cm}^{2}$ full power BL-OSL). Luminescence was detected through a $7.5 \mathrm{~mm}$ thick U-340 filter. The thermoluminescence (TL) was detected trough a combination of 7-59 and HA-3 Filters. 


\section{TL and OSL measurements}

The OSL measurements were as following. After irradiation, the disc was preheated for 5 seconds at $275^{\circ} \mathrm{C}$. Then, it was firstly stimulated by infrared light at $60^{\circ} \mathrm{C}$ for 100 s (IR-OSL) and then stimulated by blue light at $125^{\circ} \mathrm{C}$ for $100 \mathrm{~s}$ (post-IR BL-OSL or BL-OSL). All measurements were recorded by integrating one second per channel (100 channels in total).

For the TL measurement, after a preheat to $220^{\circ} \mathrm{C}$, the disc was directly cooled to $60^{\circ} \mathrm{C}$ and then heated to $650^{\circ} \mathrm{C}$ for $\mathrm{TL}$ signal measurements. The disc was then annealed at $650^{\circ} \mathrm{C}$ for 2 minutes before measuring the blackbody emission. The heating was applied at a rate of $5 \%$ under nitrogen flow. All measurements were recorded by integrating one degree per channel (650 channels in total).

\section{Method to investigate the anomalous fading}

The method was SAR-fading protocol as suggested by Auclair et al. (2003). The luminescence intensity measurement was repeated for the same irradiation dose (ca. 12 Gy) for many times (66 cycles for TL and 64 cycles for OSL). For some cycles (one every eleven cycles for TL and one every sixteen cycles for OSL), a storage time of 2 hours (long storage) was inserted between preheat and measurement. For the other cycles, we use a prompt measurement. The prompt storage time was $130 \mathrm{~s}$ in TL, $150 \mathrm{~s}$ in IR-OSL and $270 \mathrm{~s}$ for BL-OSL immediately after beta irradiation. The difference between the values for TL and IR-OSL was due to the preheat durations. For BL-OSL, the elapsed time included the time for IR-OSL measurement. g-value (percent per decade of time) was calculated using the ratio of delayed luminescence to prompt luminescence.

\section{Limits of detection and null hypothesis}

A non-fading sample is actually a sample with fading rate below the limit of detection. The limit of detection was taken as three times the standard deviation within repeatability conditions. If we excluded the cycles with large storage, the other cycles agreed with the repeatability conditions which included: same measurement procedure, same observer, same measuring instrument, used under the same conditions, same location and repetition over a short period of time. Usually, with the SAR fading method, the large number of cycles was enough to reduce the standard deviation within repeatability conditions to ca. $0.2 \%$. The limit of detection corresponded to a value of $0.6 \%$ /decade.

g-value was calculated using the delayed measurements which were periodically repeated. To control the absence of all periodic effect outside the storage, pseudo g-values were calculated for prompt measurements. Our observation given an average for the null-hypothesis (mean of the pseudo fading rate values) of $0.0 \pm 0.2 \% /$ decade. This value agreed with the observation on the repeatability.

\section{Distribution of the g-value}

Fig. 1 shows a histogram of g-values measured in our laboratory for the last three years. Negative g-values were counted as zero. Not surprisingly, IR-OSL (4-6\%/decade) shows larger values than TL and BL-OSL ( $0-4 \% /$ decade). IR-OSL is only due to feldspars while TL is a mixture of signals from both quartz and feldspars. The presence of anomalous fading in post-IR BL-OSL confirms that IR stimulation of $100 \mathrm{~s}$ is not enough to bleach all feldspars traps (Lai and Brückner, 2008).

\section{ERROR RANGE}

Fig. 2 displays the evolution of luminescence loss with time using mean g-values shown in Fig. 1 and Huntley and Lamothe's correction. We observe the drastic underestimation in the age estimation using IR-OSL signal.

For BL-OSL and TL, the luminescence loss on archaeological duration (below $50 \mathrm{ka}$ ) is obviously weaker. Although g-values are similar (Fig. 1), the losses are different for TL and BL-OSL due to the reference time. Indeed, for OSL SAR, the storage between irradiation and measurement is relatively short, ca. $270 \mathrm{~s}$ using Riso TL/OSL DA-15 and post-IR OSL protocol, including

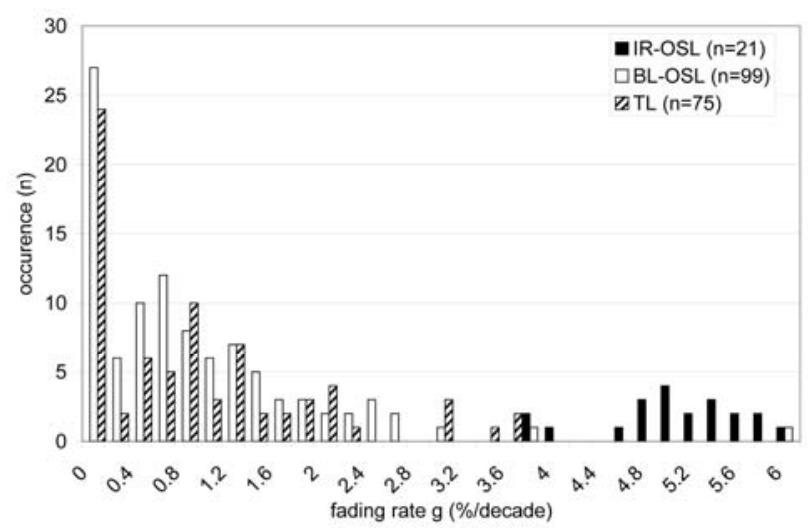

Fig. 1. g-values distribution for TL, IR-OSL and BL-OSL.

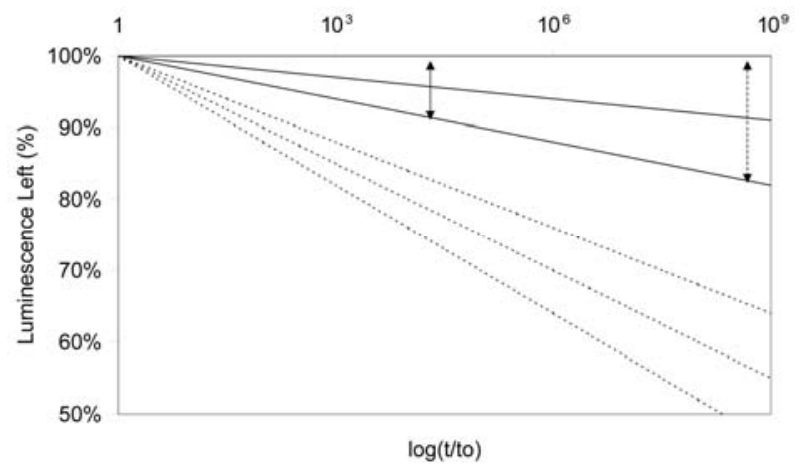

Fig. 2. Luminescence loss as function of the decade of time. The slopes correspond to $g$-values. Solid lines, $g$-values for TL or BL-OSL are equal to 0,1.0 and 2.0\%/decade. Dashed lines, $g$-values for IROSL are equal to 4.0, 5.0,6.0\%/decade. The reference time (t0) is 1 month for additive TL, $270 \mathrm{~s}$ for SAR post-IR BL-OSL and $150 \mathrm{~s}$ for SAR IR-OSL. The double arrows indicate the underestimation range for an age of $10 \mathrm{ka}$ for TL (solid line) and BL-OSL (dashed line). 
preheat treatment. Assuming an age of $10 \mathrm{ka}$, due to the amount of fading occurring during natural irradiation, the "time elapsed since irradiation" is equal to the time elapsed from a short irradiation occurring $10 \mathrm{ka} / \exp (1)=$ 3679 years ago (Aitken, 1985). The decade (of time) being a factor of 10 in "time elapsed since irradiation", the gap between $10 \mathrm{ka}$ and $270 \mathrm{~s}$ is equal to $\log (3679 \mathrm{a} / 270 \mathrm{~s})=8.6$ decades (of time). Hence, a low g-value of $1 \%$ /decade corresponds to an underestimation of $8.6 \%$. For additive TL, the storage time is more important. Usually in our laboratory, TL measurement is made one month after the end of the irradiation. In this case, the gap compared to $10 \mathrm{ka}$, is reduced to $\log (3679 \mathrm{a} / 1 \mathrm{~m})=$ 4.6 decades. g-value of $1 \% /$ decade measured for prompt time of $130 \mathrm{~s}$ corresponds to g-value of $1.04 \%$ /decade for one month (Huntley and Lamothe, 2001) and the underestimation is of $4.8 \%$ on the accumulated dose.

\section{UNCERTAINTY ESTIMATION}

The previous calculation in section 3 gives an evaluation of the range of underestimation for usual polymineral fine grain samples due to anomalous fading.

When g-value can be measured and corrections following Huntley and Lamothe (2001) used to estimate the age, it would be interesting to evaluate more precisely the uncertainties associated with the anomalous fading. Huntley and Lamothe's correction is introduced as a non linear correction:

$$
T=T f\left[1-g \cdot \log \left(\frac{T}{\exp (1) \cdot t c}\right)\right]
$$

where:

$\mathrm{T}$ is the true age and $\mathrm{Tf}$ the equivalent age measured with measurements made a time tc after the laboratory irradiations.

An estimation of the uncertainties can be found by calculating the second order term of the power series expansion. An alternative method is to use a Monte Carlo simulation method. The basic algorithm is the following (Kacker et al., 2006, Buck et al., 1996):

1) The age equation including Huntley and Lamothe's correction is developed. All input variables are associated with their probability density function (PDF).

2) Generate $M$ simulated pseudorandom numbers (q1(r)...qN(r)) for $\mathrm{r}=1$ to $\mathrm{M}$, with $\mathrm{N}$ is the number of input variables.

3) Using the cumulative probability functions (CDF) deduced from the PDFs, the M pseudorandom numbers are transformed into $\mathrm{M}$ simulated samples (Q1(r)...QN(r)).

4) Calculate the $M$ simulated age Age $(r)=f(Q 1(r) \ldots Q N(r))$

5) Calculate the estimate age (arithmetic mean of Age(r) distribution) and its standard uncertainties.

Fig. 3 shows a simulation with typical values for BLOSL measurement and g-value of $1.0 \pm 0.2 \% /$ decade. The difference between the corrected and the uncorrected ages gives the underestimation. The uncertainty on the underestimation is evaluated from the quadratic difference
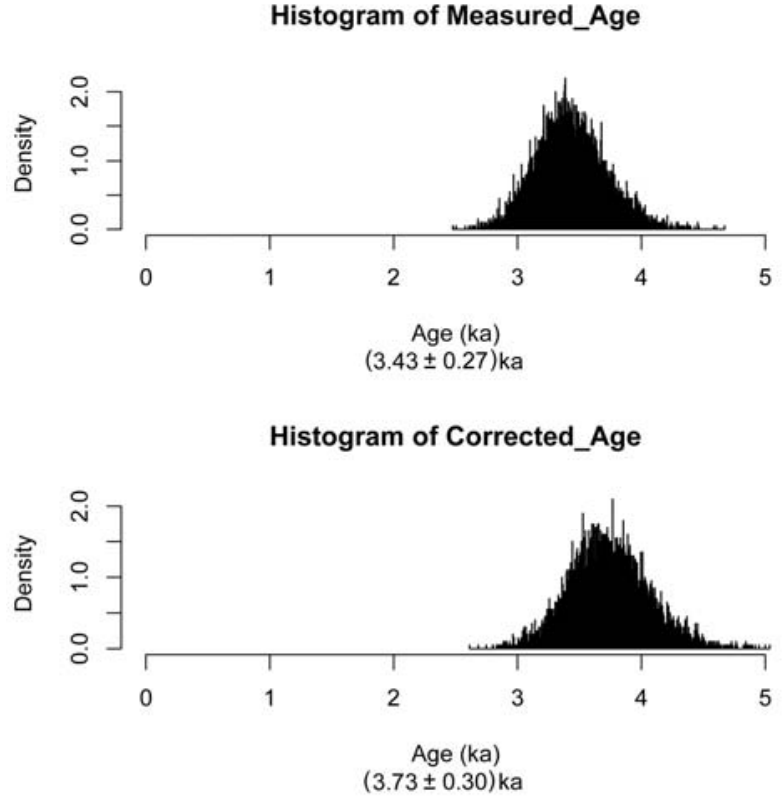

Fig. 3. Monte Carlo simulation (10000 draws) of the uncorrected (top) and corrected ages (bottom) with the input variables: equivalent dose $=10 \pm 0.5 \mathrm{~Gy}$, a-value $=0.065 \pm 0.005$, uranium content $=3.0 \pm 0.1 \mathrm{ppm}$, thorium content $=10.0 \pm 0.5 \mathrm{ppm}$, potassium oxide content $=1.00 \pm 0.05 \%$, cosmic dose $=0.15 \pm 0.02 \mathrm{~Gy} / \mathrm{ka}$, moisture content $=8.0 \pm 2.0 \%$, conversion factors from Adamiec and Aitken (1998) with 5\% uncertainties, moisture correction parameters from Zimmerman (1971) with 5\% uncertainties, storage time $=5$ minutes and $g$-value $=1.0 \pm 0.2 \% /$ decade. Calculation using $R$ language v.2.5.1 (R Development Core Team, 2007)) and rv package v.0.946 (Kerman and Gelman, 2006).

between the standard deviations for the corrected and uncorrected ages. Here, in Fig. 3, the underestimation is 300 years with an uncertainty of 130 years, or $8.0 \pm 3.5 \%$ of the true age.

\section{CONCLUSIONS}

g-values for polymineral fine grains are mainly below $3 \% /$ decade for TL and BL-OSL, and between 4 and $6 \% /$ decade for IR-OSL.

Using Huntley and Lamothe's correction for young sample $(<50 \mathrm{ka})$, measured g-values $(\mathrm{g}=\mathrm{ca} .1 \%)$ suggest an underestimation of $5 \%$ on $10 \mathrm{ka}$ for additive TL with a storage of one month after irradiation. For SAR BL-OSL, because the storage time is shorter (ca. $270 \mathrm{~s}$ ), the underestimation on $10 \mathrm{ka}$ for the same g-value increases to $9 \%$.

For the non-fading samples, with fading rates below the limit of detection $(<0.6 \%)$, the underestimation on the age for $10 \mathrm{ka}$ is below $3 \%$ in TL and 5\% in BL-OSL.

It is possible to estimate the uncertainty contribution due to anomalous fading for a sample by using a Monte Carlo method. The inference on the dating shows that the uncertainty on the anomalous fading can be a significant term of the combined uncertainty on the age, even for low fading rates.

In absence of fading investigation, such values must be used to estimate the underestimation and the uncertainty contributions due to the anomalous fading. 


\section{REFERENCES}

Adamiec G and Aitken MJ, 1998. Dose-rate conversion factors: update. Ancient TL 16: 37-49.

Aitken MJ, 1985, Thermoluminescence dating. London, Academic Press: $360 \mathrm{pp}$.

Auclair M, Lamothe M and Huot S, 2003. Measurement of anomalous fading for feldspar IRSL using SAR. Radiation measurements 37(4-5): 487-492, DOI 10.1016/S1350-4487(03)00018-0.

Buck CE, Cavanagh WG and Litton CD, 1996. Bayesian approach to interpreting archaeological data. Chichester, John Wiley and sons: $382 \mathrm{pp}$

Huntley DJ and Lamothe M, 2001. Ubiquity of anomalous fading in Kfeldspars and the measurement and correct. Canadian Journal of Earth Science 38(7): 1093-1106, DOI 10.1139/cjes-38-7-1093.

Kacker R, Toman B and Huang D, 2006. Comparison of ISO-GUM, draft GUM Supplement 1 and Bayesian statistics using simple linear calibration. Metrologia 43: S167-S177, DOI 10.1088/0026$1394 / 43 / 4 / \mathrm{S} 02$
Kerman J and Gelman A, 2006. Bayesian Data Analysis using R. Rnews 6: $21-24$.

Lai Z-P, Brückner H, 2008. Effects of feldspar contamination on equivalent doses and the shape of growth curves for OSL of siltsized quartz extracted from Chinese loess. Geochronometria 30: 49-53, DOI 10.2478/v10003-008-0010-0.

Preusser F, 2003. IRSL dating of K-rich feldspars using the SAR protocol: Comparison with independent age control. Ancient TL 21: 17 23.

R Development Core Team, 2007. R: A language and environment for statistical computing. R Foundation for Statistical Computing, Vienna, Austria. ISBN 3-900051-07-0, URL http://www.Rproject.org.

Visocekas R, 1988. Comparison between tunnelling afterglows following alpha and beta irradiation. Nuclear Tracks and Radiation Measurements 14: 163-166, DOI 10.1016/1359-0189(88)90058-1.

Winlte AG, 1973. Anomalous fading of thermoluminescence in mineral samples. Nature 245(5421): 143-144, DOI 10.1038/245143a0.

Zimmerman DW, 1971. Thermoluminescence dating using the fine grains from pottery. Archaeometry 13(1): 29-52, DOI 10.1111/j.1475-4754.1971.tb00028.x. 